\section{Clinical Enzymology}

Rudolph AbDerhalden, M.D. Translated by Peter OESPER, PH.D. Pp. xvi +448 , illustrated. Princeton, New Jersey, New York, Toronto and London: D. Van Nostrand Co. Inc. 196r. \$9.75. $76 s$.

An immense amount of work must have gone into compiling this book, which contains over 1,500 references. Perusal of the bibliography, however, shows that, with two exceptions from the German literature, no paper published after 1957 is considered. At the time of publication, therefore, the book was probably at least four years out of date, a delay which depreciates the value of the work and which must have been due to the time involved in translation. The reader seeking recent information will therefore be disappointed in the book. On the other hand, the vast number of facts assembled will be useful to those who wish to study the history of the subject and developments up to the middle of the last decade.

The book is divided into four chapters. The first, 'Fundamental of Enzymology', sets out in a concise form the essential facts about the nature and properties of enzymes. There follows a long chapter headed 'Enzymatic Pathology and Diagnosis by Enzymatic Methods', in which a large number of enzymes is discussed from the standpoints of their physiology, methods of assay and clinical significance. Assay methods are described too briefly to help the laboratory worker, who will have to study the original papers. The sections on clinical significance could profitably have been subdivided to facilitate reference to different diseases. The third and fourth chapters are entitled 'Congenital Enzyme Defects' and 'Enzyme Therapy' respectively and each contains useful information.

The volume is clearly printed on semi-gloss paper. The translator has succeeded in making the English text readable and remarkably free from Americanisms. The index could have been more comprehensive for what will be primarily a reference book.

\section{Principles of First Aid for the Injured}

H. Proctor, M.B., B.S., F.R.C.S.E. and P.S. London, M.B.E., M.B., B.S., F.R.C.S. Pp. xiii +244 , illustrated. London: Butterworths. 1962. 35 s.

The authors have made an excellent attempt to explain the disorders of function which result from an injury. The principles of treatment are laid down for the non-medical first aid enthusiast, the general practitioner who is interested in first aid and the accident surgeon. The last mentioned in particular should find this book interesting because of the exposition of the modern ideas of shock and the organization of an accident service, which begins at the site of the accident.

The common lethal effects of injury are loss of blood, serious damage to the chest and suffocation. They are enumerated in the second chapter and are repeated at intervals throughout the book. The first aider is given explicit advice on what to do and what not to do. The chapter ends with an interesting account of the modern views on drowning and the crush syndrome.

There is a fascinating account of the various aspects of shock and a lucid description of accurate methods of assessment of the degrees of shock. The management of fractures, dislocations and minor injuries are adequately dealt with and the management of burns is comprehensive. The mechanism of production of head injuries is summarised and unconsciousness is explained as a disturbance of the reticular formation. Care of the airway is the most important measure in the first aid of cranio cerebral injuries.

There is an excellent account of the disordered physiology of chest injuries. The first aider is told how: he can help patients with paradoxical respiration and an open pneumothorax. The dangers inherent in first $\stackrel{\text { ? }}{+}$ aid for spinal injuries are stressed in order to enable the non-medical practitioner to play a useful role.

Finally, sensible advice is given on the disposal of patients at the site of the accident.

\section{Casualty Officer's Handbook}

MaUrice Ellis, M.A., M.B., B.ChIR(CANTAB.), $\overrightarrow{0}$ F.R.C.S.(ENG.), L.R.C.P., D.T.M. and H. Pp. vii + 243, illustrated, London: Butterworths. 1962. 42s. $\vec{\omega}$

This book supplies most of the answers to the्ठ problems of diagnosis and management which confront the junior Casualty Officer when he is faced with patient and has no one to turn to for immediate guidance. $\omega_{\infty}$ A method of treatment is described, which may be applicable in the hospital in which he works.

The book begins with the management of skeletal and soft tissue trauma. Symptoms and signs are presented in a clear and lifelike way. Attention is $\vec{t}$ drawn to common pitfalls and the interpretation of $x$-rays is simplified. The author's management of a? sprained ankle is novel and interesting but the Casualty_ Officer may run up against a shortage of facilities for physiotherapy in his own hospital. The managemento of minor fractures and common dislocations is described and the author's results should be good because of his 3 obvious attention to detail.

A concise description is given of the management $8 f$ minor wounds, the method of tetanus prevention and $\overrightarrow{0}$ the possible role of Penicillin as a prophylactic age Burns which require admission are separated from those which can be successfully treated in the Casualty Department. Burns have been treated successfully as outpatients by the open method but the author has reserved a place for the closed method. The author's treatmento of abscesses is based on a study of their pathology buto the Casualty Officer should consult his chief before suturing abscesses after drainage. Sound advice is given on the treatment of foreign bodies.

The description of acute appendicitis is delightful and the other acute abdominal emergencies are adequately dealt with. The role of release tenderness ando absence of bowel sounds in the diagnosis of ruptured abdominal viscera is not stressed but it seems churlish to look for blemishes in an otherwise excellent book.

\section{Fundamental Techniques of Plastic Surgery}

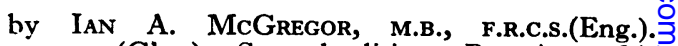
F.R.F.P.S.(Glas.). Second edition. Pp. xi +286, illustrated. Edinburgh and I.ondon: E. \& S. Livingstone. 1962. 32s. $6 d$.

The appearance of a second edition of this book within two years of its original publication is ample
testimony to its popuiarity.

No major changes have been incorporated by the $\mathrm{N}$ author, beyond the addition of an excellent, simple andN well-illustrated chapter on maxillo-facial injuries.

Criticism may reasonably be levelled at the work on the grounds of a certain lack of balance and incompleteco ness. For example the neuro-vascular island flap, of which a brief description is given, can hardly be described as a fundamental technique and is unlikely to be undertaken by other than a fully trained plastic 